\title{
Broadband Radar Absorbing Structures with a Practical Approach from Design to Fabrication
}

\author{
Won-Ho Choi ${ }^{1} \cdot$ Woon-Hyung Song ${ }^{1} \cdot$ Won-Jun Lee ${ }^{2, *}$
}

\begin{abstract}
In this study, a novel broadband radar absorbing volume structure (RAVS) is proposed and demonstrated with a practical point of view from design to fabrication. The proposed RAVS uses a design concept of repeatedly stacked carbon nanotube (CNT) composites and foam cores of the same thickness to improve the applicability to real structures while maintaining absorption performance. The repeatedly stacked CNT composites, which act as electrically lossy materials, result in the multiple scattering of incident electromagnetic waves trapped inside the structure. The trapped incident waves then lose their energy by multiple scattering. Based on this design concept, the RAVS designed through field analysis and parametric study achieved a $-10 \mathrm{~dB}$ absorption performance from $4 \mathrm{GHz}$ to $16 \mathrm{GHz}$. With reference to the design values, RAVS was fabricated for verification, and the absorption performance was measured using a free space measurement system. The measurement result showed excellent absorption performance that satisfied $-10 \mathrm{~dB}$ from $5.8 \mathrm{GHz}$ or less to 14 GHz.
\end{abstract}

Key Words: Low Observable Aircraft, Multi-Functional Composites, Radar Absorbing Structure, Smart Structures.

\section{INTRODUCTION}

A radar absorbing structure (RAS) is a multifunctional composite structure that simultaneously has load-bearing and electromagnetic (EM) wave absorption functions. The goal of RASs is to reduce the radar cross-section (RCS) of the target and increase its survivability [1].

In recent years, RASs have been actively researched and implemented in various forms with various types of absorbing materials. Although previously reported RASs can effectively reduce RCS through their unique absorbing materials and/or by changing the lay-up structure to optimize the absorption performance [2-12], most of the proposed RASs have been focused on the X-band (8.2-12.4 GHz).
When the radar absorber is designed for a narrowband application and radar frequencies other than the design frequency, the design values deviate from the optimal values, significantly degrading the absorption performance. Moreover, the performance of narrowband designs is sensitive to fabrication tolerance. Therefore, a robust design considering the changes in radar frequencies and fabrication tolerance is required.

The Jaumann absorber is the representative broadband absorbing structure $[1,13-15]$. It is a multilayered structure that consists of dielectric spacers and resistive sheets. Although the Jaumann absorber can perform broadband absorption, the dielectric thickness and resistance of each layer should have different design values for optimal performance. Despite its excellent absorption performance, such a structure is burdensome at the

Manuscript received April 6, 2020 ; Revised May 18, 2020 ; Accepted July 16, 2020. (ID No. 20200406-041J)

${ }^{1} \mathrm{R} \& D$ Center, Korean Air, Daejeon, Korea.

${ }^{2}$ The 7th R\&D Institute-2, Agency for Defense Development, Daejeon, Korea.

"Corresponding Author: Won-Jun Lee (e-mail: ngwljys@gmail.com)

This is an Open-Access article distributed under the terms of the Creative Commons Attribution Non-Commercial License (http://creativecommons.org/licenses/by-nc/4.0) which permits unrestricted non-commercial use, distribution, and reproduction in any medium, provided the original work is properly cited.

(c) Copyright The Korean Institute of Electromagnetic Engineering and Science. All Rights Reserved. 
time of fabrication because there are many design parameters to be controlled. In addition, although the absorption performance in the planar structure is excellent, the absorption performance on a planar structure cannot be fully achieved when applied to curved structures [16]. Choi and Kim [17] reported a broadband radar absorbing honeycomb structure with a design concept that uses the transverse direction of the hexagonal passages to increase the effective thickness. The researchers have presented a design concept that utilizes the entire volume of a leadingedge structure. However, when the full-depth honeycomb core is applied to such a leading-edge shape, the additional composite materials should be laminated for fastening with other parts. In this case, the bonding strength at the interface between the honeycomb core and the additional laminated composite is likely to be weak because of the small contact area. Although the proposed broadband radar absorbing honeycomb structure shows an outstanding absorption performance, there is limited applicability to the leading-edge shape, which is essential for reducing RCS over a wide frequency range.

To resolve such issues, this study proposes a broadband radar absorbing volume structure (RAVS) that uses the entire volume of the structure for EM wave absorption. To improve applicability and manufacturability, the proposed RAVS uses a structure in which the carbon nanotube (CNT) composites and foam cores are repeatedly stacked with the same thickness. The repeatedly stacked CNT composites, which act as electrically lossy materials, trap the multiple scattering of incident EM waves inside the structure. The trapped incident waves then lose their energy by multiple scattering.

Fig. 1 shows the application concept of the proposed RAVS using the leading-edge shape. As shown in Fig. 1(a), first, a core block in which foam cores and CNT composites are repeatedly stacked is fabricated, and the fabricated core block is machined according to the shape to be applied. Finally, the machined core block is stacked with other composite materials for autoclave cure. Fig. 1(b) shows the final form of the RAVS applied to the leading-edge shape. As shown in Fig. 1(b), because the interface between the core block and the composite shear web is bonded face-to-face, the bonding strength can be increased compared with the radar absorbing honeycomb core.

\section{MATERIAL PREPARATION}

To design a broadband RAVS, CNT prepreg, foam core, and glass fiber reinforced polymer (GFRP) were prepared. Here, CNT prepreg refers to the prepreg coated on the glass fabric with CNT-dispersed epoxy resin. The role of a CNT is to increase the loss tangent of resin. The CNT is a multi-walled design purchased from Carbon Nanotech, Pohang, Korea. Carbon purity was greater than $95 \%$, and the average diameter was 15

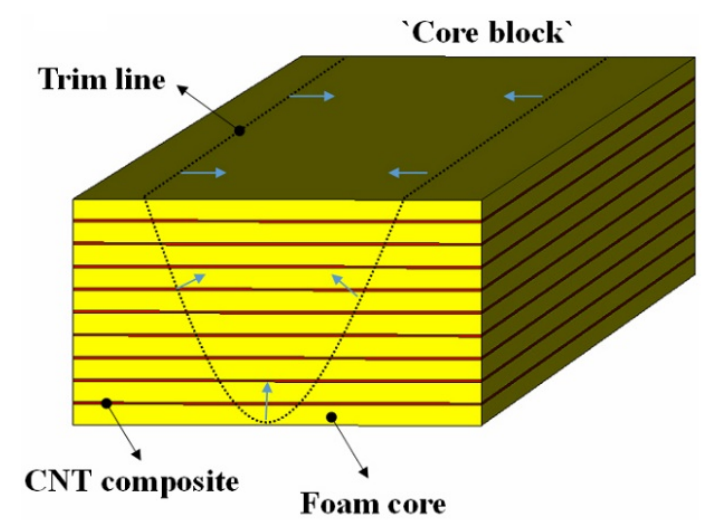

(a)

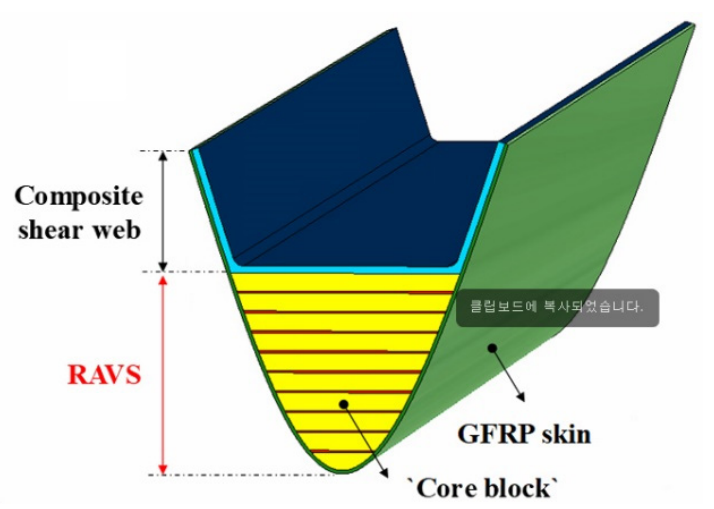

(b)

Fig. 1. Schematic diagram of the application concept of the RAVS: (a) fabrication of the core block and (b) final form of the RAVS in a leading-edge shape.

nm. To fabricate the CNT prepreg, the prepared CNT powder was dispersed in epoxy resin using a planetary mixer. The final CNT content of the mixed constituent material was $3.0 \mathrm{wt} \%$. Using a prepreg manufacturing machine, the mixed constituent materials were coated on a dry glass fabric. The GFRP and foam core used for the RAVS were purchased from $\mathrm{Cytec}(\mathrm{Cy}-$ com), Brussels, Belgium and Evonik (Rohacell), Essen, Germany, respectively.

A free space measurement system was used to measure the permittivity of the prepared composite materials $[18,19]$. The free space measurement system consists of two spot-focusing horn antennas, a sample holder, and a network analyzer. The role of the spot-focusing horn antennas is to minimize measurement inaccuracies caused by scattering at the edge of the specimens. By replacing the focusing horn antennas with different frequency bands, the system can measure in the frequency range of 5.8-18 GHz. The through-reflect-line (TRL) calibration technique and time-domain gating were used to eliminate undesirable reflections. The free space measurement system was controlled using a computer connected with a general-purpose interface bus cable. 
Fig. 2 shows the measurement results for the broadband permittivity characteristics. As shown in Fig. 2(a) and (b), the permittivity of the GFRP and foam core did not significantly depend on the frequencies. However, in the case of the CNTcomposite, the change in permittivity exhibited a frequency de-

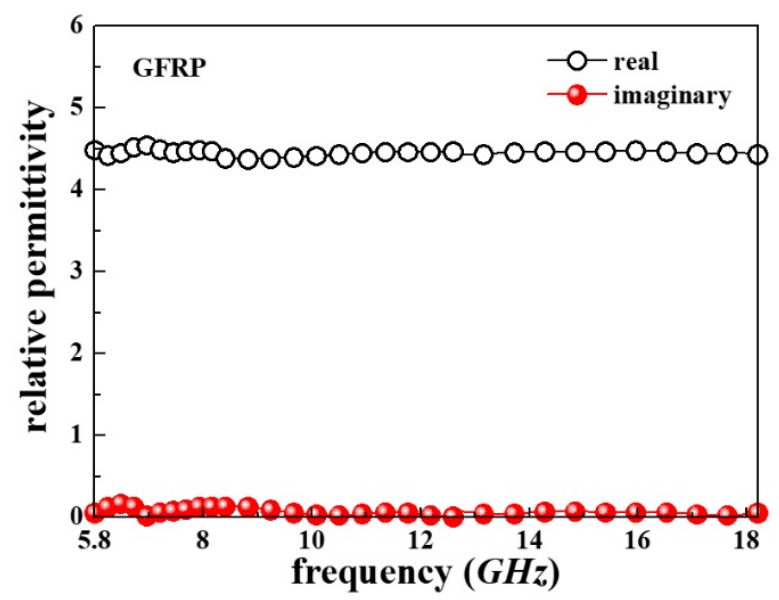

(a)

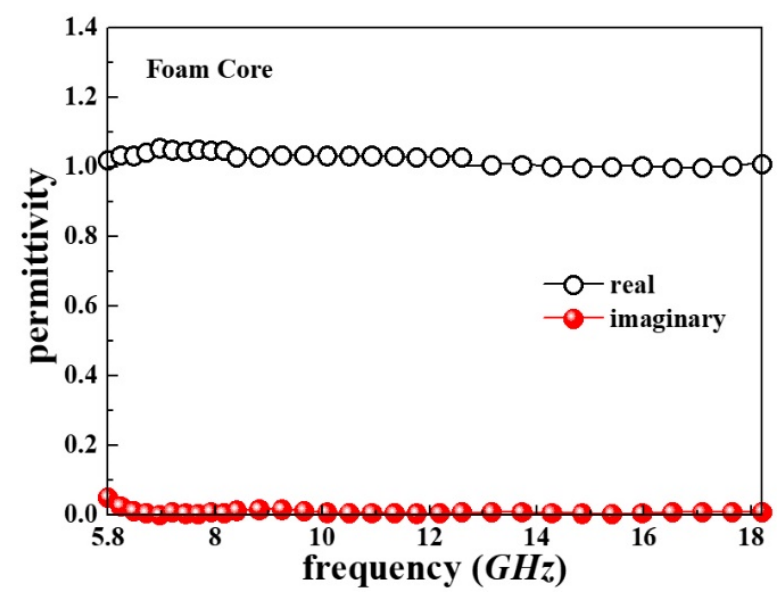

(b)

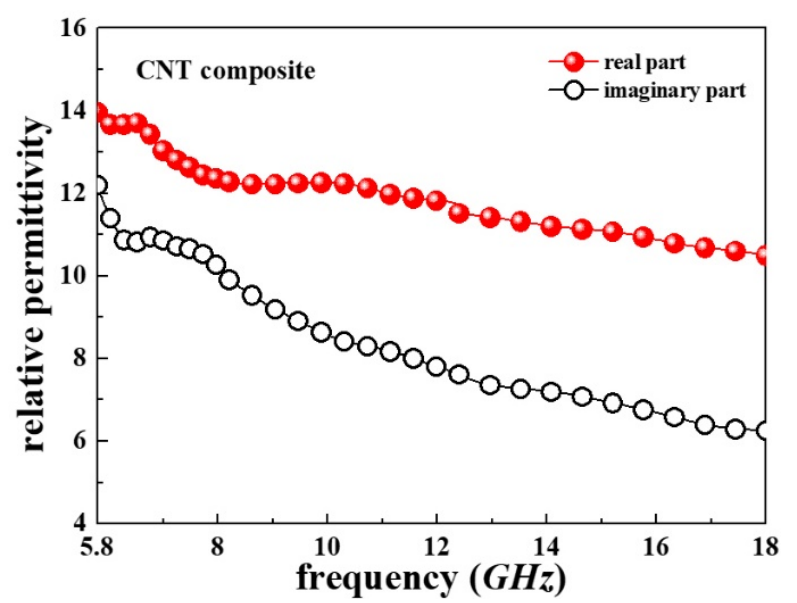

(c)

Fig. 2. Permittivity measurement results: (a) GFRP, (b) foam core, and (c) CNT composite. pendence due to the effects produced by the dispersion of the conductive CNT fillers in epoxy resin.

\section{DESIGN PRINCIPLES}

To design a radar absorber, the theoretical relationship between thickness and material properties should be understood. Rozanov and his colleagues $[20,21]$ reported the theoretical bandwidth limitations of radar absorbers for a given thickness and set of material properties. In the case of a multi-layer absorber, the largest possible bandwidth for a given physical thickness and permeability can be written as:

$$
\left|\ln \rho_{o}\right| \Delta \lambda<2 \pi^{2} \sum_{j} \mu_{s, j} d_{j}
$$

where $\rho_{o}$ is the desired reflection coefficient, $\Delta \lambda$ is the bandwidth defined by $\Delta \lambda=\lambda_{\max }-\lambda_{\min }\left(\lambda_{\max }\right.$ and $\lambda_{\text {min }}$ are the wavelengths at the lower and upper band edge, respectively), and $\mu_{u, j}$ and $d_{j}$ are the static permeability and thickness at each layer, respectively. As described in Eq. (1), to enlarge the bandwidth of the radar absorber, the permeability and thickness of each layer should be large. Generally, magnetic materials are beneficial for implementing broadband absorbing performance. However, in the case of the radar absorber using magnetic materials, a large number of magnetic particles should be impregnated in the matrix for a sufficient absorption performance in the high-frequency ranges. This approach is accompanied by a weight increase. Moreover, because having too many magnetic particles negatively impacts the mechanical properties, magnetic absorbers are not suitable as a load-bearing composite structure [22]. However, in the case of non-magnetic materials, according to Eq. (1), the absorption bandwidth is only controlled by thickness; that is, if the total thickness increases, the weight increases. Therefore, to minimize the weight increase, a lowdensity foam core was used as the primary material.

For the design of the RAVS, the transmission line Eq. (1) was used and is written as Eqs. (2) and (3) as follows:

$$
\begin{gathered}
\eta_{n+1}=\eta_{n} \frac{\eta_{n-1}+j \eta_{n} \tan \left(k_{n} d_{n}\right)}{\eta_{n}+j \eta_{n-1} \tan \left(k_{n} d_{n}\right)} . \\
\rho=\frac{\eta_{n+1}-\eta_{o}}{\eta_{n+1}+\eta_{o}} .
\end{gathered}
$$

Using Eqs. (2) and (3), the input impedance and reflection coefficient of the multilayered structures were calculated. Here, $\eta_{n+1}$ is the input impedance on the surface of the absorber; $\eta_{n}$ and $\eta_{n-1}$ are the impedances in the $n^{\text {th }}$ and $n-1^{\text {st }}$ layer, respectively; $k_{n}$ is the wave number in the $n^{\text {th }}$ layer; and $d_{n}$ is the thickness in the $n^{\text {th }}$ layer.

Unlike conventional radar absorbers, the proposed design concept uses the attenuations of EM waves by multiple scattering. Fig. 3 shows the principles of the design concept of the 


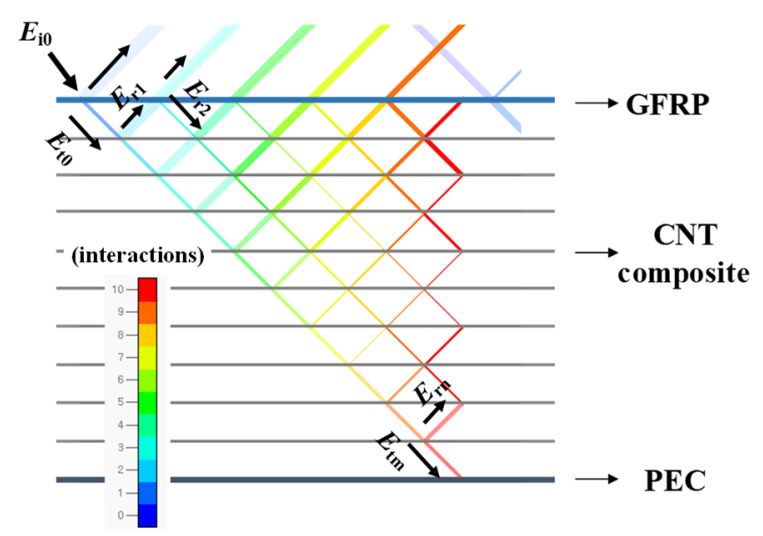

(a)

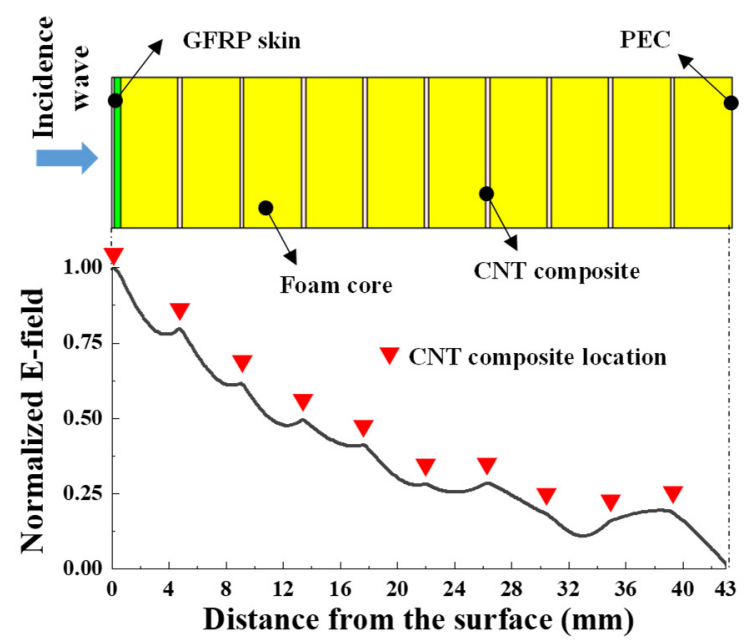

(b)

Fig. 3. Principles of the RAVS: (a) ray interactions and (b) field intensity distribution.

RAVS through ray interaction analysis and field analysis. Note that although Fig. 3(a) illustrates the case of oblique incidence differently from Fig. 3(b), this is only for convenience of explanation because the ray trajectory is visible at the oblique incidence. In Fig. 3(a) when the incident wave impinges on the surface of the RAVS, the EM waves are trapped inside the RAVS. These trapped EM waves proceed to lose their energy by successive transmissions and reflections by the repeatedly stacked CNT composites. As a result, the field intensity decreases as the EM waves propagate from the surface to the bottom of the RAVS as shown in Fig. 3(b). Here, the small peaks denoted in the field intensity graph as triangle symbols indicate the presence of reflections and transmissions due to the difference in the wave impedance of the foam core and the CNT composite.

As shown in Figs. 1 and 3, the proposed RAVS has a structure stacked with the same thickness of CNT composites and foam cores. Such a design approach is different from the design concept of the conventional RAS in which the thickness of each layer is different for an optimal design. As the RAVS has few design variables, it has the advantage of design simplicity and hence manufacturability.

\section{DESIGN OF THE RAVS}

Fig. 4 shows the schematic diagram of the initial design configuration of the RAVS. Note that the number of CNT composite layers is $\mathrm{N}-1$, where $\mathrm{N}$ is the number of foam cores.

First, to determine the design range, the absorption bandwidth for the change in thickness of the CNT composite and foam core was analyzed (Fig. 5).

Fig. 5(a) shows the $-10 \mathrm{~dB}$ bandwidth for the change in thickness of the CNT composite and foam core. The target zone was set to a bandwidth greater than $10 \mathrm{GHz}$. However, there was no information on which frequency band the $-10 \mathrm{~dB}$ bandwidth should cover. Therefore, as the X-band is the most important frequency band for RCS reduction, the return loss for the design variables within the target zone at $10 \mathrm{GHz}$ was analyzed as shown in Fig. 5(b). The return loss distribution shows that the best performance for the foam core thickness at 10 $\mathrm{GHz}$ was approximately $4 \mathrm{~mm}$. To determine the number of foam cores, the return loss was analyzed depending on the number of foam core layers. Fig. 6 shows the return loss vs. the number of foam core layers at each frequency. As shown in the figure, the return loss converges at more than 10 layers of foam cores.

Return loss convergence can also be confirmed in the field distribution depending on the number of foam core layers. To simulate the field distribution in the RAVS, CST Microwave Studio [23] was used.

Fig. 7(a) shows the analysis unit-cell model of the RAVS

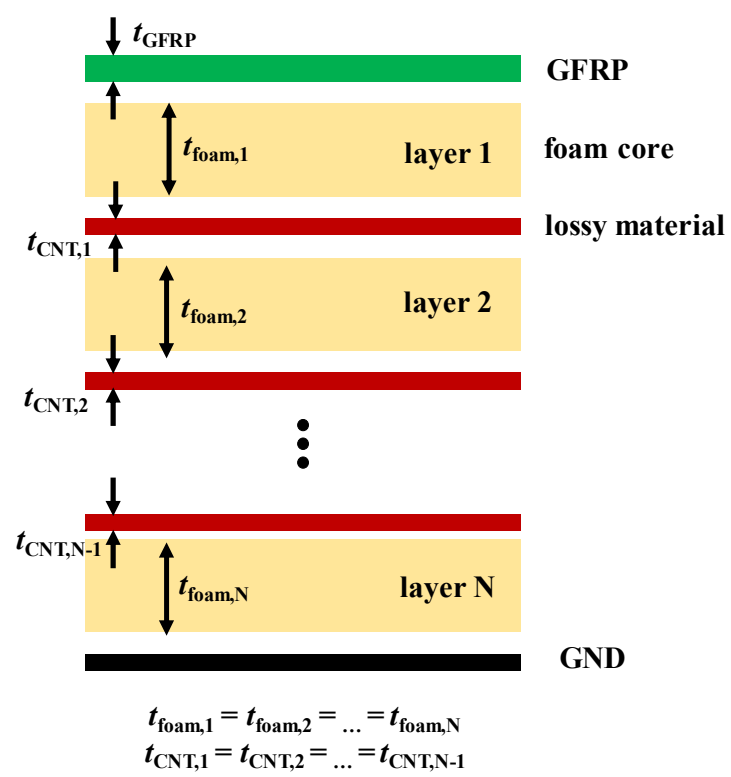

Fig. 4. Initial configuration for the design of the RAVS. 


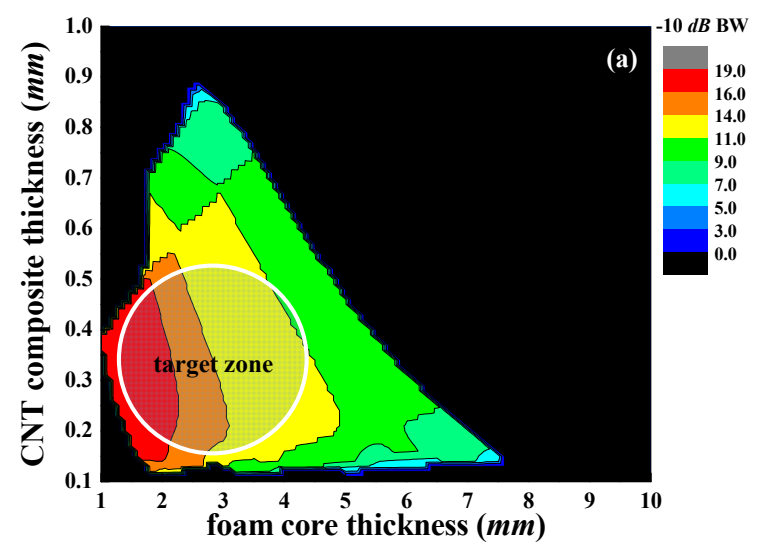

(a)

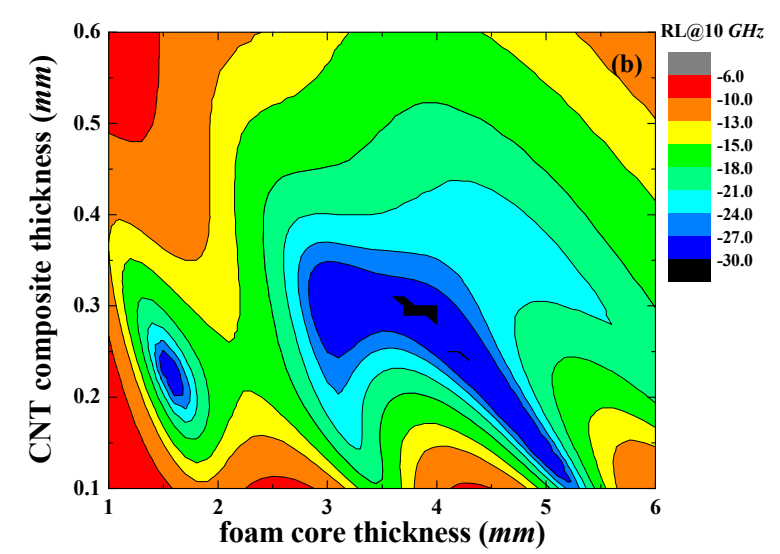

(b)

Fig. 5. Design procedures of the RAVS: (a) setup for the design target zone and (b) selection of design parameters.

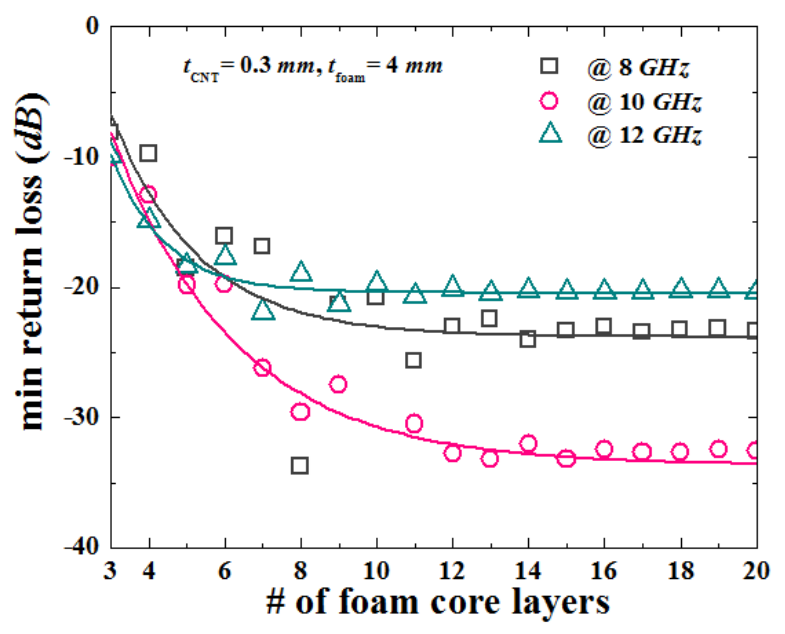

Fig. 6. Change in absorption performance vs. the number of foam core layers.

with boundary conditions. Tangential fields, $\mathrm{E}_{\mathrm{t}}$ and $\mathrm{H}_{\mathrm{t}}$, were set to 0 for the periodic boundary characteristic of the unit-cell. As shown in Fig. 7(b), compared with the foam cores with 7 and 13 layers, the foam cores with 10 layers were sufficient for the attenuation of field intensity. As the absorption mechanism of

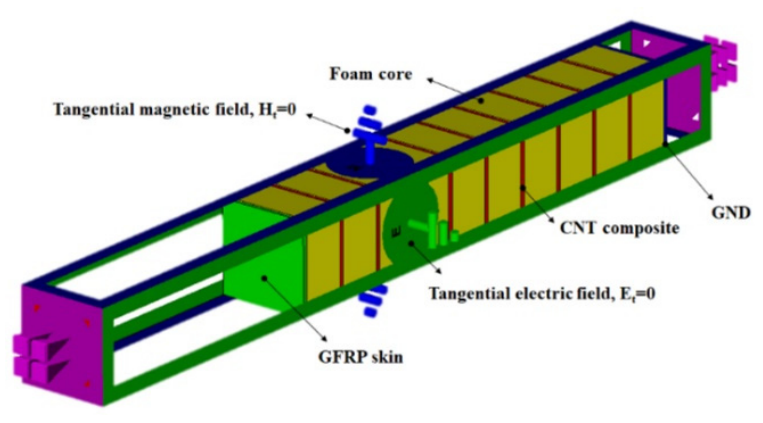

(a)

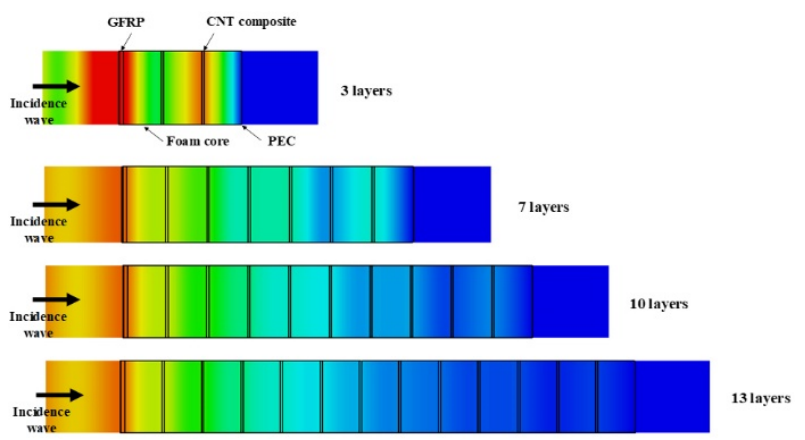

(b)

Fig. 7. (a) Simulation model for field analysis with a boundary condition and (b) a field distribution depending on the number of foam core layers.

the RAVS is field attenuation trapped inside the structure by multiple scattering, no additional layers were found to be necessary when the RAVS was designed with sufficient layers. With reference to the analysis results, the thicknesses of the foam core and CNT composite were determined to be $4 \mathrm{~mm}$ and $0.3 \mathrm{~mm}$, respectively, and the total numbers of foam cores and CNT composites were set to 10 and 9, respectively. In the following section, the proposed RAVS was verified through fabrication and measurement using the determined design parameters.

\section{FABRICATION AND MEASUREMENT OF THE RAVS}

The fabrication process flow for the RAVS is summarized in Fig. 8. With reference to the design values, the foam cores were machined to the designed thickness using a composite trim router. The thickness tolerance of the machined foam cores was less than $0.15 \mathrm{~mm}$. In the lay-up step, two plies of GFRP were laid up on the tool, and then the CNT prepregs and foam cores were repeatedly stacked. As a ground material, a flexible copper clad laminate was laid up for fabrication simplification. After finishing the lay-up step, curing accessories, such as a nonperforated release film, breather, and bagging film, were placed onto the stacked RAVS specimen. The layered RAVS specimen was cured in an autoclave with the curing accessories for 120 minutes at $130^{\circ} \mathrm{C}$. The cured specimen was cut to the di- 
(a)
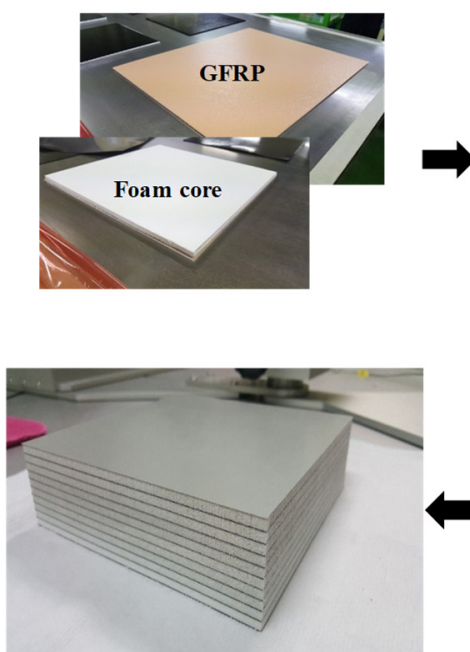

(f) (b)
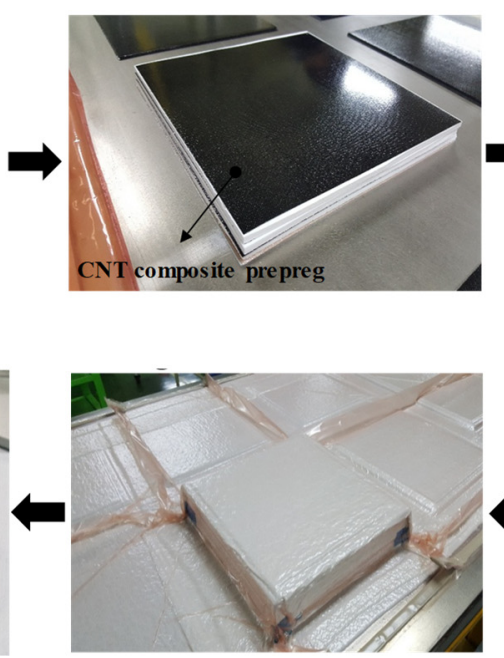

(e) (c)

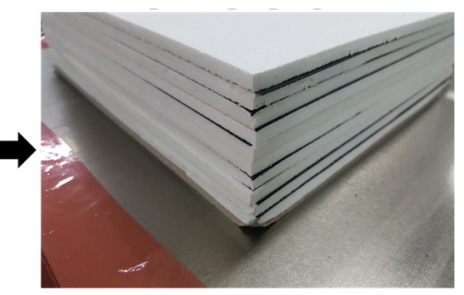

$\downarrow$

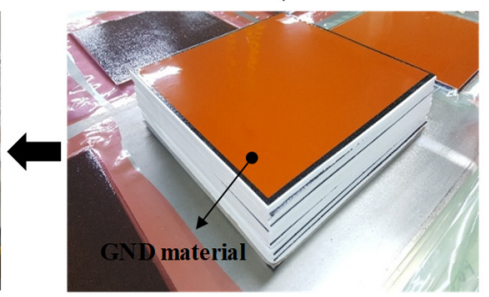

(d)

Fig. 8. Fabrication process flow of the designed RAVS: (a) GFRP and foam core lay-up, (b) CNT composite prepreg, (c) repetition of foam core and CNT composite prepreg, (d) lay-up of ground material, (e) vacuum bagging with curing accessories, and (f) autoclave cured specimen.

mensions of $180 \mathrm{~mm} \times 180 \mathrm{~mm}$ to measure its absorption performance.

The absorption performance of the fabricated specimen was measured using the free space measurement system. Fig. 9(a) shows the free space measurement system with spot-focusing

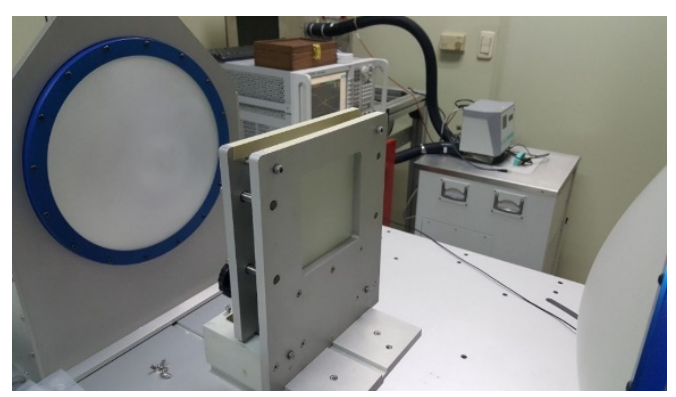

(a)

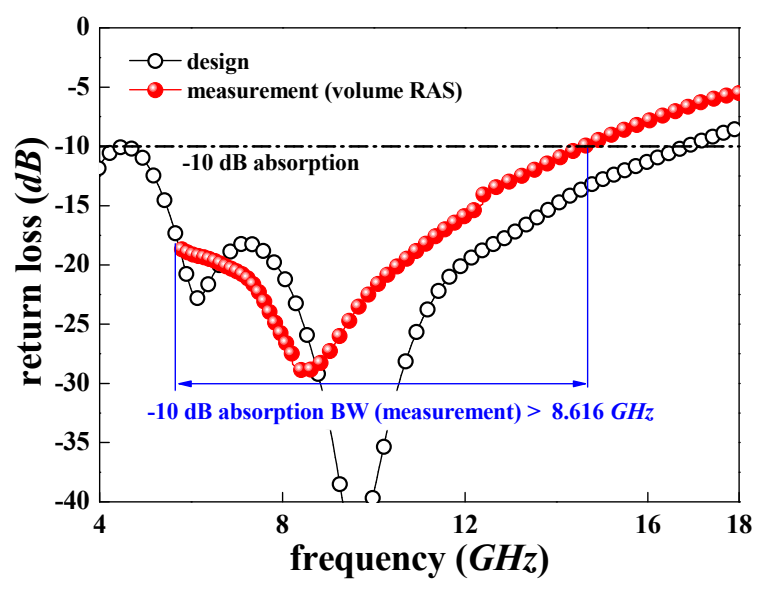

(b)

Fig. 9. (a) Free space measurement system and (b) return loss measurement result of the fabricated specimen. horn antennas and a specimen inserted into the sample holder. In Fig. 9(b), the results of the measurement of absorption performance and the simulation in the frequency range of 5.8-18 $\mathrm{GHz}$ were compared. As shown in Fig. 9, the overall tendency of the measurement result was in good agreement with the simulation result, even though there was a small shift in the frequency at approximately $1 \mathrm{GHz}$ from the simulation result due to the permittivity variation depending on the dispersibility of the CNT in epoxy. Furthermore, the fabricated RAVS satisfied the $-10 \mathrm{~dB}$ absorption bandwidth at frequencies greater than 8.6 GHz. The proposed RAVS showed excellent absorption performance in terms of the absorption bandwidth. Although the proposed RAVS has a smaller absorption bandwidth of about $5 \mathrm{GHz}$ than previously reported broadband radar absorbers [17], which utilize similar application concepts, the proposed RAVS is meaningful in that it improved the applicability by simplifying the structure and proposed a specific application concept.

\section{CONCLUSION}

This study proposes a broadband RAVS to improve applicability and manufacturability. The proposed RAVS has a stacked structure of CNT composites and foam cores of the same thickness to simplify the structure by minimizing the design variables. The absorption mechanism of the RAVS is explained using multiple scattering by the CNT composite walls. The repeatedly stacked CNT composites, which act as electrically lossy materials, trap the multiple scattering of incident EM waves inside the structure. The trapped incident waves then lose their energy by multiple scattering. As an application concept of the RAVS, 
a core block is machined into the shape to be applied. The proposed RAVS has a smaller absorption bandwidth than previously reported broadband radar absorbers. However, it has an absorption performance of $-10 \mathrm{~dB}$ or more in the C-band and $\mathrm{X}$-band, which are important frequency bands at the radar frequency. From a practical point of view, this study provides an important concept and application method for the RAVS.

This research was conducted with the support of the Leading Defense Core Technology R\&D projects (No. UC160032JD) by the Agency for Defense Development.

\section{REFERENCES}

[1] K. J. Vinoy and R. M. Jha, Radar Absorbing Materials: From Theory to Design and Characterization. Boston, MA: Kluwer Academic Publishers, 1996.

[2] J. B. Kim and J. H. Byun, "Salisbury screen absorbers of dielectric lossy sheets of carbon nanocomposite laminates," IEEE Transactions on Electromagnetic Compatibility, vol. 54, no. 1, pp. 37-42, 2012.

[3] W. J. Lee, J. W. Lee, and C. G. Kim, "Characteristics of an electromagnetic wave absorbing composite structure with a conducting polymer electromagnetic bandgap (EBG) in the X-band," Composites Science and Technology, vol. 68, no. 12, pp. 2485-2489, 2008.

[4] S. E. Lee, J. H. Kang, and C. G. Kim, "Fabrication and design of multi-layered radar absorbing structures of MWNTfilled glass/epoxy plain-weave composites," Composite Structures, vol. 76, no. 4, pp. 397-405, 2006.

[5] H. K. Jang, W. H. Choi, C. G. Kim, J. B. Kim, and D. W. Lim, "Manufacture and characterization of stealth wind turbine blade with periodic pattern surface for reducing radar interference," Composites Part B: Engineering, vol. 56, pp. 178-183, 2014.

[6] K. Y. Park, S. E. Lee, C. G. Kim, and J. H. Han, "Fabrication and electromagnetic characteristics of electromagnetic wave absorbing sandwich structures," Composites Science and Technology, vol. 66, no. 3-4, pp. 576-584, 2006.

[7] W. H. Choi, J. B. Kim, J. H. Shin, T. H. Song, W. J. Lee, Y. S. Joo, and C. G. Kim, "Circuit-analog (CA) type of radar absorbing composite leading-edge for wing-shaped structure in X-band: practical approach from design to fabrication," Composites Science and Technology, vol. 105, pp. 96-101, 2014.

[8] J. H. Oh, K. S. Oh, C. G. Kim, and C. S. Hong, "Design of radar absorbing structures using glass/epoxy composite containing carbon black in X-band frequency ranges," Composites Part B: Engineering, vol. 35, no. 1, pp. 49-56, 2004.
[9] Y. W. Nam, J. H. Choi, W. J. Lee, and C. G. Kim, "Thin and lightweight radar-absorbing structure containing glass fabric coated with silver by sputtering," Composite Structures, vol. 160, pp. 1171-1177, 2017.

[10] Y.W. Nam, J. H. Choi, W. J. Lee, and C. G. Kim, "Fabrication of a thin and lightweight microwave absorber containing Ni-coated glass fibers by electroless plating," Composites Science and Technology, vol. 145, pp. 165-172, 2017.

[11] W. H. Choi, J. H. Shin, T. H. Song, J. B. Kim, W. Y. Lee, and C. G. Kim, "A thin hybrid circuit-analog (CA) microwave absorbing double-slab composite structure," Composite Structures, vol. 124, pp. 310-316, 2015.

[12] G. Kim, S. Kim, and B. Lee, "Design of wideband microwave absorbers using reactive Salisbury screens with maximum flat reflection," Journal of Electromagnetic Engineering and Science, vol. 19, no. 2, pp. 71-81, 2019.

[13] L. J. Du Toit, "The design of Jauman absorbers," IEEE Antennas and Propagation Magazine, vol. 36, no. 6, pp. 1725, 1994.

[14] L. J. Du Toit and J. H. Cloete, "Electric screen Jauman absorber design algorithms," IEEE Transactions on Microwave Theory and Techniques, vol. 44, no. 12, pp. 2238-2245, 1996.

[15] B. Chambers and A. Tennant, "Optimised design of Jaumann radar absorbing materials using a genetic algorithm," IEE Proceedings-Radar, Sonar and Navigation, vol. 143, no. 1, pp. 23-30, 1996.

[16] P. Y. Ufimtsev, "Comments on diffraction principles and limitations of RCS reduction techniques," Proceedings of the IEEE, vol. 84, no. 12, pp. 1830-1851, 1996.

[17] W. H. Choi and C. G. Kim, "Broadband microwaveabsorbing honeycomb structure with novel design concept," Composites Part B: Engineering, vol. 83, pp. 14-20, 2015.

[18] D. K. Ghodgaonkar, V. V. Varadan, and V. K. Varadan, "A free-space method for measurement of dielectric constants and loss tangents at microwave frequencies," IEEE Transactions on Instrumentation and Measurement, vol. 38, no. 3, pp. 789-793, 1989.

[19] D. K. Ghodgaonkar, V. V. Varadan, and V. K. Varadan, "Free-space measurement of complex permittivity and complex permeability of magnetic materials at microwave frequencies," IEEE Transactions on Instrumentation and Measurement, vol.39, no. 2, pp.387-394, 1990.

[20] K. N. Rozanov, "Ultimate thickness to bandwidth ratio of radar absorbers," IEEE Transactions on Antennas and Propagation, vol. 48, no. 8, pp. 1230-1234, 2000.

[21] K. N. Rozanov, A. V. Osipov, D. A. Petrov, S. N. Starostenko, and E. P. Yelsukov, "The effect of shape distribution of inclusions on the frequency dependence of permeability in composites," Journal of Magnetism and Magnetic Materials, vol. 321, no. 7, pp. 738-741, 2009. 
[22] W. Gao, X. Wang, and W.Xu, "Magneto-mechanical properties of polydimethylsiloxane composites with a binary magnetic filler system," Polymer Composites, vol. 40, no. 1,

\section{Won-Ho Choi}

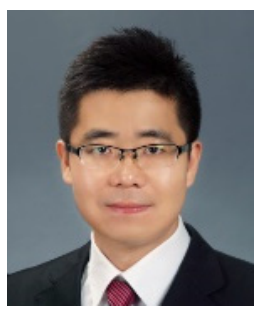

received a Ph.D. in aerospace engineering from the Korea Advanced Institute of Science and Technology (KAIST), Daejeon, Korea, in 2014. From 2015 to 2017, he was a Senior Researcher at Hyundai Heavy Industries, Yongin, Korea. He has been a Deputy General Manager in the Research and Development Center at Korean Air since 2017. In 2008, he was an intern researcher at SEMATECH, Austin, TX, US. His current research interests include microwave-absorbing materials for far-field/near-field waves, electromagnetic applications of composite materials, nanocomposites, and nanotechnology. pp. 337-345, 2019.

[23] CST Studio Suite [Online]. Available: https://www.3ds. com/products-services/simulia/products/cst-studio-suite.

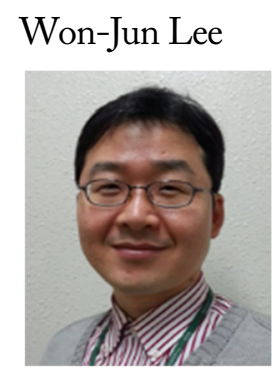

received a $\mathrm{Ph} . \mathrm{D}$. in aerospace engineering from the Korea Advanced Institute of Science and Technology (KAIST), Daejeon, Korea, in 2008. In 2008, he was with SK Innovation, Daejeon, Korea where he was involved in the development of battery packaging for automobile application. He joined the Agency for Defense Development, Daejeon, Korea, in 2012. His current position is Senior Researcher. His current research interests include microwave-absorbing structures, low observable radomes, and the structural design of composite materials for aerospace applications.

\section{Woon-Hyung Song}

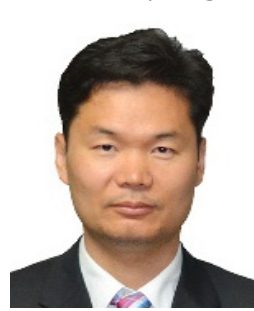

received a master's degree in aerospace engineering from the Korea Advanced Institute of Science and Technology (KAIST), Daejeon, Korea, in 2000. In 2000, he joined the R\&D center for Korean Air, Daejeon, Korea where he was involved in the development of composite structures for satellites and aircraft structures. His current position is Deputy General Manager. His current research interests include radar absorbing structures, frequency selective surface radomes, and the structural design of composite materials for aerospace applications. 